\title{
Bases Moleculares da Glomerulopatia Diabética
}

\begin{abstract}
RESUMO
O principal determinante da nefropatia diabética é a hiperglicemia, mas hipertensão e fatores genéticos também estão envolvidos. O glomérulo é o foco de lesão, onde proliferação celular mesangial e produção excessiva de matriz extracelular decorrem do aumento da glicose intracelular, por excesso de glicose extracelular e hiperexpressão de GLUT1. Seguem-se aumento do fluxo pela via dos polióis, estresse oxidativo intracelular, produção intracelular aumentada de produtos avançados da glicação não enzimática (AGEs), ativação da via da PKC, aumento da atividade da via das hexosaminas e ativação de TGF- $\beta 1$. Altas concentrações de glicose também aumentam angiotensina II (All) nas células mesangiais por aumento intracelular da atividade da renina (ações intrácrinas, mediando efeitos proliferativos e inflamatórios diretamente). Portanto, glicose e All exercem efeitos proliferativos celulares e de matriz extracelular nas células mesangiais, utilizando vias de transdução de sinais semelhantes, que levam a aumento de TGF- $\beta 1$. Nesse estudo são revisadas as vias que sinalizam os efeitos da glicose e All nas células mesangiais em causar os eventos-chaves relacionados à gênese da glomerulopatia diabética. As alterações das vias de sinalização implicadas na glomerulopatia, aqui revisadas, suportam dados de estudos observacionais/ensaios clínicos, onde controle metabólico e anti-hipertensivo, especificamente com inibidores do sistema renina-angiotensina, têm-se mostrado importantes - e aditivos - na prevenção do início e progressão da nefropatia. Novas estratégias terapêuticas dirigidas aos eventos intracelulares descritos deverão futuramente promover benefício adicional.

(Arq Bras Endocrinol Metab 2007;51/6:901-912)
\end{abstract}

Descritores: Nefropatias diabéticas; Patogênese; Mecanismos moleculares de ação

\section{ABSTRACT}

Molecular Bases of Diabetic Nephropathy.

The determinant of the diabetic nephropathy is hyperglycemia, but hypertension and other genetic factors are also involved. Glomerulus is the focus of the injury, where mesangial cell proliferation and extracellular matrix occur because of the increase of the intra- and extracellular glucose concentration and overexpression of GLUT1. Sequentially, there are increases in the flow by the poliol pathway, oxidative stress, increased intracellular production of advanced glycation end products (AGEs), activation of the PKC pathway, increase of the activity of the hexosamine pathway, and activation of TGF- $\beta 1$. High glucose concentrations also increase angiotensin II (AII) levels. Therefore, glucose and All exert similar effects in inducing extracellular matrix formation in the mesangial cells, using similar transductional signal, which increases TGF- $\beta 1$ levels. In this review we focus in the effect of glucose and All in the mesangial cells in causing the events related to the genesis of diabetic nephropathy. The alterations in the signal pathways discussed in this review give support to the observational studies and clinical assays, where metabolic and antihypertensive controls obtained with angiotensin-converting inhibitors have shown important and additive effect in the prevention of the beginning and progression of diabetic nephropathy. New therapeutic strategies directed to the described intracellular events may give future additional benefits. (Arq Bras Endocrinol Metab 2007;51/6:901-912)

Keywords: Diabetic nephropathy; Pathogenesis; Molecular mechanisms of action revisão

\section{Claudia J. Lagranha \\ PATRICIA FIORINO \\ DulCe Elena CASARINI \\ BEATRIZ D'AGORD SCHAAN \\ MARIA Claudia IRIGOYen}

Laboratório de Hipertensão

Experimental, Unidade de

Hipertensão, Instituto do

Coração, HC-FMUSP (CJL, PF \&

$\mathrm{MCl}$ ), e Laboratório de

Nefrologia, Disciplina de

Nefrologia, Escola Paulista de

Medicina-UNIFESP (DEC), São

Paulo, SP; e Laboratório de Cardiologia Molecular e Celular do Instituto de Cardiologia, Fundação Universitária de Cardiologia (BD'AS), Porto Alegre, RS.

Recebido em 03/07/06

Revisado em 19/01/07

Aceito em 14/05/07 
$\mathrm{O}$ DIABETES MELLITUS É UMA DOENÇA crônicodegenerativa cuja prevalência mundial em 1995 foi de $4 \%$ (135 milhões de portadores), sendo projetada para o ano de 2025 uma estimativa de 5,4\% (300 milhões de portadores), representando um aumento na incidência de 122\% (1). No Brasil, esta taxa, em 1986 , correspondeu a $7,6 \%$ da população urbana com mais de 30 anos (2), mas dados mais atualizados, especificamente do RS, mostram quase o dobro desta prevalência $(12,4 \%)(3)$, confirmando as tendências mundiais apresentadas.

Os indivíduos com diabetes têm alto risco para a doença macrovascular e esta é sua principal causa de morte (4). Diversos fatores de risco são passíveis de prevenção e tratamento, como a nefropatia diabética e a hipertensão arterial sistêmica. A nefropatia diabética acomete cerca de $40 \%$ dos pacientes com diabetes, sendo a principal causa de insuficiência renal naqueles que ingressam em programas de diálise. A mortalidade dos pacientes diabéticos em programas de hemodiálise é maior do que a dos não diabéticos. Cerca de $40 \%$ dos pacientes morrem no primeiro ano de tratamento, principalmente por doença cardiovascular (5), e o custo do tratamento de insuficiência renal crônica é elevado.

A nefropatia diabética incipiente caracteriza-se por microalbuminúria, enquanto que a nefropatia clínica se caracteriza por macroalbuminúria ou proteinúria persistentes. Nesta fase, a progressão inexorável à insuficiência renal crônica e necessidade de diálise $\mathrm{e} /$ ou transplante renal eram a regra, mas o controle metabólico intensivo (6), tratamento rigoroso da hipertensão arterial e o uso de drogas com efeito bloqueador do sistema renina-angiotensina-aldosterona (7) têm sido estratégias eficazes na redução da incidência e desaceleração da progressão da doença (8). No entanto, ainda um número considerável de pacientes desenvolve esta complicação e suas mais graves conseqüências, sendo necessária a identificação dos mecanismos envolvidos na sua gênese para se obter prevenção e tratamento mais eficientes.

O principal determinante na gênese e progressão da nefropatia diabética é a hiperglicemia (6), processo que pode ser modificado por susceptibilidade genética (9) e acelerado por outros fatores, especialmente a hipertensão arterial sistêmica (10). Os efeitos no rim ocorrem especificamente nos glomérulos, foco principal de lesão na nefropatia diabética. O diabetes seletivamente lesa células cujo transporte de glicose não se reduz com a hiperglicemia, como é o caso das células mesangiais, endoteliais da retina e neurônios de nervos periféricos, acarretando maior concentração intracelular de glicose. Vários são os mecanismos envolvidos no dano tecidual causado pela hiperglicemia: aumento do fluxo pela via dos polióis, o que aumenta a sensibilidade ao estresse oxidativo intracelular, produção intracelular aumentada de produtos avançados da glicação não enzimática (AGEs), ativação da via da PKC e aumento da atividade da via das hexosaminas (11).

Estudos histopatológicos em humanos e em animais de experimentação verificaram que o processo de esclerose dos glomérulos renais inicia-se com a proliferação de células mesangiais, que produzem excessivamente componentes da matriz extracelular, cujo depósito excessivo é a via final comum de várias doenças renais, inclusive a diabética (12). Os principais componentes da matriz extracelular são fibronectina, laminina, tenascina e colágeno (tipo IV, V e VI) (13). Entretanto, no glomérulo esclerótico, além desses componentes há também acúmulo de colágeno tipo I e tipo III, que geralmente não são detectados na matriz extracelular do glomérulo normal (14). O colágeno tipo IV é a proteína mais abundante na matriz mesangial (12) e sua deposição aumenta com a gravidade da doença glomerular (15). A tenascina, outra proteína da matriz mesangial normal, pode também ser encontrada em situações patológicas e seu acúmulo é proporcional ao grau de esclerose glomerular (16).

Conforme proposto por Brownlee, em sua Banting Lecture apresentada em 2004 (11) sobre os mecanismos patofisiológicos do diabetes, 4 principais mecanismos são desencadeados pelo aumento da glicose intracelular e a partir daí são responsáveis pela gênese das complicações crônicas do diabetes: 1) aumento do fluxo pela via dos polióis, gerando estresse oxidativo, 2) aumento dos produtos avançados da glicação não enzimática (AGEs), gerando aumento de glicação de proteínas plasmáticas e da matriz extracelular, 3) ativação da via da PKC, gerando aumento de citocinas, estresse oxidativo e fatores proliferativos, e 4) aumento do fluxo pela via das hexosaminas, gerando aumento de citocinas. $\mathrm{O}$ fato de que pacientes tratados com drogas que bloqueiam um desses mecanismos não terem como resultado a prevenção completa das complicações crônicas, assim como não haver relação aparente entre os 4 mecanismos citados, levou o autor a propor um mecanismo unificador. Este está representado pelo aumento de estresse oxidativo na mitocôndria induzido pelo aumento da glicose intracelular, o qual leva à quebra das fitas do DNA nuclear, que ativa a poli (ADP-ribose) polimerase (PARP). Esta modifica a atividade de uma enzimachave da via glicolítica, a gliceraldeído-3-fosfato 
(GADPH), reduzindo sua atividade, o que ativa a via dos polióis, aumenta a formação de AGEs, ativa a PKC e aumenta o fluxo pela via das hexosaminas. Estes mecanismos estão sumarizados na figura 1 .

\section{EVENTOS MOLECULARES DA LESÃO GLOMERULAR ASSOCIADOS À ELEVAÇÃO DA GLICOSE}

O advento e a popularização de novas metodologias ao longo das duas últimas décadas vem possibilitando um enorme avanço no conhecimento das bases moleculares de processos biológicos. Uma das conseqüências imediatas deste avanço é a unificação progressiva dos vários ramos da biologia experimental, permitindo que a biologia molecular, biologia celular, bioquímica, genética e fisiologia desenvolvam-se de maneira indistinta através de abordagens experimentais. Esta revolução nas ciências básicas nos permite identificar e caracterizar eventos moleculares que até o momento permaneciam pouco elucidados.

As células mesangiais glomerulares desempenham papel fundamental na manutenção da estrutura e funcionamento fisiológico normal dos glomérulos. Estudos in vitro utilizando células mesangiais cultivadas em meios com alta concentração de glicose demonstraram maior expressão de mRNA e síntese protéica de colágeno tipo IV e fibronectina (17),

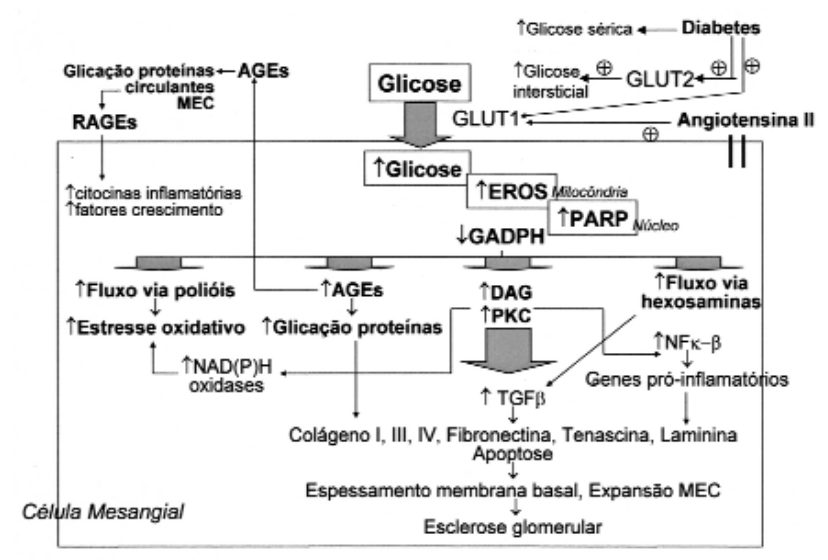

Figura 1. Representação esquemática das vias através das quais a hiperglicemia, decorrente do diabetes, causa aumento de glicose intracelular e subseqüentemente glomeruloesclerose.

AGEs: Produtos avançados de glicação não-enzimática; RAGEs: receptores para os AGEs; EROS: Espécies reativas de oxigênio; PARP: Poli(ADP-ribose) polimerase; GADPH: gliceraldeído-3-fosfato; DAG: Diacilglicerol; PKC: proteína quinase $C$; MEC: matriz extra-celular. alterações que são mediadas pela ativação da PKC (18). A glicose entra para o interior da maioria das células, inclusive as mesangiais, por difusão facilitada. Este processo é mediado por proteínas integrais de membrana específicas, os transportadores de glicose (GLUTs) (19). Estudos com células mesangiais in vitro demonstraram maior expressão de GLUTl em meios com alta concentração de glicose, maior atividade da PKC e conseqüente estímulo à síntese de proteínas da matriz extracelular (20).

Estudos de nosso grupo demonstraram, in vivo, no modelo de diabetes por STZ em ratos, maior expressão de GLUTl em córtex renal, associado à maior excreção urinária de TGF- $\beta 1$ e microalbuminúria (21). O GLUT2, principal transportador responsável pela reabsorção tubular de glicose, também se encontra expresso no diabetes experimental (22), favorecendo aumento da glicose intersticial, a qual é transportada então para o interior das células mesangiais pela ação do GLUTl (figura 1). No modelo animal de hipertensão geneticamente determinada (ratos SHR) com diabetes por STZ, os resultados foram semelhantes, além de se observar um efeito aditivo da hipertensão sobre a hiperglicemia em aumentar a expressão de GLUT1 e GLUT2 (21). Estudos in vitro identificaram o GLUTl como o principal transportador de glicose nas células mesangiais de camundongos (23) e humanos (24). Um transporte de glicose aumentado através da membrana celular pode, portanto, amplificar os efeitos da hiperglicemia característica do diabetes, contribuindo para a gênese da glomerulopatia diabética.

Conforme Leehey e cols. (25), em sua revisão, experimentos in vitro com células mesangiais demonstraram que o aumento na expressão de GLUTl induz a uma retroalimentação positiva, aumentando ainda mais a expressão de GLUT1, ativando a PKC, promovendo uma estimulação prejudicial da síntese de matriz extracelular em células mesangiais. Com isso, a elevação na expressão de GLUTl parece ser o passo limitante para o uso de glicose pelas células mesangiais.

Nose e cols. (26) foram os primeiros a demonstrar que a angiotensina II (AII) aumenta a expressão e o acúmulo de RNA mensageiro (RNAm) de GLUT1 em células mesangiais. Estes autores também mostraram que o efeito estimulador da AII é principalmente mediado pelo processamento e liberação da heparin-binding epidermal growth factor-like growth factor (HB-EGF) via ativação das metaloproteinases (MMP) com ligação ao receptor (EGF-R), seguido da ativação da via de sinalização da ERK. Previamente, estudos desse mesmo grupo (27) já haviam demons- 
trado que a ativação de ERK mediava a indução da expressão de fibronectina estimulada pela AII em células mesangiais, e que a fosforilação de ERK ocorre depois da ativação do receptor de EGF pela ligação da heparina ao receptor. Nesse mesmo estudo, verificouse que a transativação do EGF-R era mediada pelo receptor ATl para AII.

Estudos mostram que as células mesangiais possuem todos os componentes do sistema renina-angiotensina (SRA), incluindo renina, angiotensinogênio, receptor tipo 1 de AII (ATl), ECA somática e ECA N-domínio $(28,29)$, sugerindo a existência de um SRA intra-renal que parece ser regulado independentemente do SRA convencional presente na circulação sangüínea (30). Conforme Leehey e cols. (25) sugerem, as células mesangiais podem não necessitar de ECA para a síntese de AII, e podem utilizar outras peptidases para converter a angiotensina I (AI) em AII. Além das células mesangiais, todos os componentes do SRA foram detectados em células epiteliais do túbulo proximal (31), células endoteliais glomerulares (32) e células do ducto coletor (33). Em especial, sabe-se que a ECA N-domínio encontra-se localizada no núcleo das células mesangiais em cultura primária e é responsável pela transcrição de novos genes (34).

As células mesangiais são capazes de sintetizar fatores que promovem sua mitogênese in vitro, como a interleucina I (IL-I) (35) e o fator de crescimento semelhante ao fator de crescimento derivado de plaquetas. Efeitos mitogênicos também são descritos na presença do fator de crescimento derivado de plaquetas (PDGF), insulina (36), EGF (epidermal growth factor), TGF- $\beta$, prostaglandina $\mathrm{F}_{2 \alpha}\left(\mathrm{PGF}_{2 \alpha}\right)$ e endotelina (37).

A ação do TGF- $\beta$ depende de sua concentração: em pequenas concentrações, é mitogênico, mas em altas concentrações inibe a proliferação celular, as prostaglandinas, nucleotídeos cíclicos e o tromboxano $\mathrm{A}_{2}$, que possuem atividades anti-mitogênicas sobre a cultura de células mesangiais. Alguns constituintes da própria matriz extracelular exercem efeitos sobre as células mesangiais: a fibronectina, uma glicoproteína de adesão, promove a proliferação celular (38), enquanto os proteoglicanos inibem o crescimento das células mesangiais (39), sugerindo que alguns elementos da matriz possuam um papel na regulação do crescimento celular. De fato, Righetti e cols. (40) mostraram acúmulo precoce de fibronectina em córtex renal de animais SHR tornados diabéticos.

Estudos in vitro mostram que meio de cultura com alta concentração de glicose exerce influência sobre o crescimento de células renais e sobre o cres- cimento da matriz extracelular, de forma semelhante ao que ocorre in vivo (41). Além disto, altas concentrações de glicose em culturas de células mesangiais de ratos aumentam a produção de AII (42).

Vidotti e cols. (29) demonstraram que, em condições basais, as células mesangiais secretam prórenina, mas na presença de altas concentrações de glicose ocorre um decréscimo desta secreção. Após um tempo de 72 horas de exposição das células mesangiais ao meio contendo alta concentração de glicose, somente o RNA mensageiro da ECA e angiotensinogênio estão elevados. Esses resultados sugeriram que altas concentrações de glicose induzem aumento da geração de AII nas células mesangiais em decorrência do aumento intracelular da atividade da renina mediado por três fatores: estimulação tempo-dependente da transcrição do gene da pró-renina, redução da secreção da pró-renina e aumento da conversão da pró-renina em renina ativa, mediado pela catepsina $B$.

O aumento do RNA mensageiro do angiotensinogênio em paralelo ao aumento da atividade da renina indica que a alta concentração de glicose também aumenta os níveis do substrato para a renina, sugerindo que, ao lado da renina, a ECA está diretamente envolvida no aumento da AII nas células mesangiais. A produção de AII pelas células mesangiais sob estimulação da glicose faz com que este hormônio tenha ações intrácrinas mediando os efeitos proliferativos e inflamatórios diretamente, com conseqüente proliferação celular, expansão de matriz e inflamação, contribuindo diretamente com a esclerose glomerular observada na nefropatia diabética (29).

Como vimos acima, ambas, glicose e angiotensina II, parecem exercer efeitos similares sobre células mesangiais em cultura, promovendo o crescimento celular, proliferação da matriz extracelular e inibindo a sua degradação (29). Possivelmente glicose e AII utilizam semelhantes vias de transdução de sinais, que em última análise levam a um aumento da produção celular de TGF- $\beta$ (32).

O aumento de glicose em culturas de células ativa mecanismos e vias que levam ao aumento da transcrição do TGF- $\beta 1$ em células mesangiais; entre as conhecidas está a ativação da via MEK-ERK (43) e a ativação da PKC. Além do aumento de glicose no meio de cultura, sabe-se que a AII também tem efeito estimulador da transcrição e ativação do TGF- $\beta 1$ (44). Uma vez ativado nas células mesangiais, o TGF- $\beta 1$ pode determinar aumento na apoptose dessas células (45), da proliferação celular (46), da formação e deposição de proteínas de matriz como laminina, fibronectina e colágeno do tipo I e do tipo IV $(47,48)$, 
diminuição da degradação das proteínas de matriz por aumento da expressão de PAI-1 (inibidor do ativador de plasminogênio-1), induzindo assim esclerose glomerular $(37,43)$.

Os mecanismos pelos quais o aumento de glicose no meio de cultura pode induzir disfunção nas células mesangiais, e outros tipos celulares, envolvem aumento da síntese autócrina de fatores de crescimento (TGF- $\beta 1$, AII, fator de crescimento derivado de plaquetas e endotelina 1) ou alteração das vias de sinalização como a da PKC (49). Esses fatores de crescimento, além de poder promover diretamente a alteração de vias de sinalização intracelulares, estimulam a produção de espécies reativas de oxigênio (ROS), que por sua vez podem atuar como segundo mensageiro (50). Além desses efeitos, o aumento de glicose no meio de cultura promove alteração na sinalização de $\mathrm{Ca}^{2+}$ nas células mesangiais. Mené e cols. (51) demonstraram que o influxo de cálcio estava reduzido em meios com alta concentração de glicose. Seguindo essa mesma linha de estudo, Hua e cols. (52) demonstraram que a elevação de glicose no meio suprime a sinalização de $\mathrm{Ca}^{2+}$ em resposta à endotelina 1 , e sugerem que esse efeito está associado à modulação das sub-unidades da PKC (PKC- $\beta$ e PKC- $\delta$ ) e da produção de ROS pelas células mesangiais.

$\mathrm{Xu} \&$ Kyriakis (53) demonstraram que AGEs em cultura de células mesangiais estimulam a fosforilação de ERK via ativação da sub-unidade K-Ras, de maneira dependente da ativação de PI3K, proteína efetora de Ras na maioria das, mas não em todas as, vias de sinalização (54). Inibidores farmacológicos de Ras têm sido extensivamente utilizados em estudos para a terapia do câncer, e pesquisadores sugerem que esses inibidores também poderiam ser úteis na prevenção de doenças renais (55).

Resultados anteriores de Danesh e cols. (56) demonstraram que as células mesangiais expostas a meios com alta concentração de glicose apresentavam maior proliferação e expressão das proteínas da família das GTPases, Ras e Rho. Nesse mesmo estudo, foi observado que o co-tratamento das células mesangiais com um inibidor da HMG-CoA redutase, a simvastatina, revertia os efeitos causados pela elevação da glicose no meio na indução de Ras e Rho e na proliferação. $\mathrm{O}$ processo de prenilação é insuficiente para a translocação e localização na membrana das isoformas de Ras, necessitando de um sinal adicional para sua ativação. Danesh e cols. (56) sugerem que o sinal adicional pode ser tanto promovido pelo farnesil como pelo geranilgeranil, que são modificações nos lípides indispensáveis pela ancoragem funcional da proteína Ras na membrana plasmática.
Outra família de proteína relacionadas às GTPases são as Arfs, que podem modular o recrutamento de proteínas quinases de membrana e ativar eventos de sinalização via interação com a PKC. Já foi demonstrado que o diabetes induz alterações no fenótipo das células mesangiais por ativação das isoformas de PKC. Padival e col. (57) demonstraram, em células mensagiais cultivadas com alta concentração de glicose no meio de cultura, aumento da presença de PKC $\beta 1$ e Arf 6 na membrana, e sugeriram que Arf 6 poderia atuar nas alterações do fenótipo das células mesangiais associadas à glomerulopatia diabética.

Lin e cols. (58), estudando outras proteínas da família das GTPases, avaliaram a indução de fibronectina pela alta glicose em células mesangiais. Neste estudo, descreveram a expressão e ativação de Rap $1 \beta$ e a importância de PKC e Raf na síntese de fibronectina, e demonstraram que a alta concentração de glicose causa ativação específica de Rap $1 \beta$, uma vez que Rap $2 \beta$ permaneceu inalterada neste ambiente. Além disso, demonstraram que Rapl $\beta$ estimula a síntese de fibronectina e que esse efeito da alta concentração de glicose na Rap e fibronectina é dependente de PKC e de Raf, e independente de PDGF, sugerindo um novo caminho de aumento de produção de proteínas de matriz estimulado pela alta concentração de glicose que é PKC-Rapl $\beta$-Raf (58).

Schnaper e cols. (59), avaliando as proteínas de matriz, demonstraram que o TGF $\beta 1$ está relacionado com o acúmulo de colágeno tipo 1 por estimulação via Smad. Em outro estudo, demonstraram que TGF $\beta 1$ rapidamente induz rearranjo do citoesqueleto das células mesangiais por aumento das fibras de estresse ( $\alpha$-actina), por promover a redistribuição e a união do complexo de adesão focal, como também por modular a proteína Rho da família das GTPase. Esses autores verificaram que a inibição de Rho por toxina B C. difficile ou por Y-27632 bloqueava o efeito deletério do TGF- $\beta 1$ na estimulação da expressão de colágeno do tipo I (60).

\section{EVENTOS MOLECULARES DA LESÃO GLOMERULAR DO DIABETES ASSOCIADOS AO ÓXIDO NÍTRICO}

Em modelos experimentais de glomerulopatia, desconsiderando-se o mecanismo de lesão renal, o óxido nítrico (NO) tem sido identificado como um potencial regulador do controle da proliferação das células mesangiais (61), o que implicaria em seu papel de modificar a deposição de matriz extracelular no mesângio, contribuindo para a progressão das anormalidades glomerulares (62). 
A importância do tempo de exposição à alta concentração de glicose, tanto em cultura de células como em modelos experimentais in vivo, são importantes no entendimento da fisiopatologia do óxido nítrico nas modificações glomerulares (63).

$\mathrm{O}$ óxido nítrico é uma molécula gasosa e reativa com uma meia-vida de poucos segundos, formado a partir do aminoácido L-arginina em uma reação de oxidação catalisada por uma família de enzimas denominadas de óxido nítrico sintases (NOS) (64). Duas destas isoenzimas são dependentes de cálcio e expressas constitutivamente nas células endoteliais (eNOS ou tipo III NOS) e nas células neuronais (nNOS ou tipo I NOS), e a expressão da terceira isoenzima (iNOS ou tipo II NOS), independente da presença de cálcio, é dependente de um estímulo imunológico ou inflamatório, podendo ser expressa em uma variedade de células (65). A ação do óxido nítrico não se dá através da utilização de receptores celulares, apenas difunde-se pela membrana celular ativando seu segundo mensageiro, guanilato ciclase, que por sua vez atua sobre proteínas-alvo ativando várias cascatas de proteínas quinases, incluindo as vias da MAPK, JAK e JNK (66). Essas respostas intracelulares desencadeiam efeitos fisiológicos importantes, como o de ser um potente vasodilatador, com importante repercussão tanto na regulação da pressão arterial quanto na função renal (67).

$\mathrm{O}$ óxido nítrico também pode reagir com espécies reativas de oxigênio (68), atuando nas vias de sinalização de fatores de crescimento, como o NGF e VEGF (69), ou reagindo diretamente com o ânion superóxido formando peroxinitrito, potente oxidante celular, envolvido na progressão da doença renal.

Na progressão do diabetes, observa-se acúmulo progressivo dos AGEs associado com a hiperglicemia (70). Estudos recentes demonstraram que os AGEs induzem a expressão da iNOS em células mesangiais,

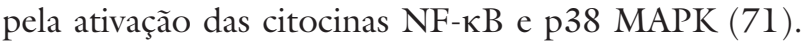
Noh e cols. (72) também demonstraram que, em cultura de células mesangiais, o ambiente rico em glicose aumentava a expressão da iNOS e que esta, por sua vez, induzia a expansão extracelular pelo aumento da deposição de fibronectina.

Em glomérulo isolado de animais diabéticos, observou-se correlação entre a ativação da PKC e a supressão do óxido nítrico. Em cultura de células mesangiais, verificou-se aumento das proteínas de matriz mediado pela PKC e redução do GMPc. O aumento da PKC relaciona-se aos níveis celulares aumentados de diacilglicerol, maior modulador endógeno das vias glicolíticas da metabolização da glicose. Em conjunto, estas observações sugerem que o prejuízo da formação do GMPc, mediado pela redução do óxido nítrico, em resposta ao aumento da $\mathrm{PKC}$ em ambiente rico em glicose, pode representar uma importante via pela qual a toxicidade da glicose é traduzida em aumento da susceptibilidade celular no diabetes (73).

Veelken e cols. (74) mostraram que, no diabetes experimental de 2 semanas induzido por estreptozotocina, o aumento da expressão e da atividade da eNOS, tanto em glomérulo quanto na arteríola aferente, seria a causa do aumento do óxido nítrico que, por sua vez, levaria à hiperperfusão glomerular, contribuindo para iniciação da nefropatia diabética. Em cultura de células mesangiais, altas concentrações de glicose estimulavam a expressão tanto do RNA mensageiro quanto da proteína da iNOS via ativação da PKC, promovendo acúmulo da matriz extracelular, corroborando as avaliações in vivo (72). Khamaisi e cols. (75) mostraram tendência ao aumento da expressão da eNOS no córtex renal após 1 semana de diabetes, mantendo tal aumento após 2 a 8 semanas. A expressão da isoforma nNOS, por sua vez, foi reduzida em $52 \%$ após 2 semanas de diabetes (75).

A fosforilação da eNOS pela PKC reduz sua atividade catalítica (76), que, por sua vez, seria acompanhada pela fosforilação da Thr495 e desfosforilação da Ser1177 (77). Tal interação entre PKC e eNOS sugere uma forte associação entre diabetes e redução da NOS renal. Por outro lado, em cultura de CMS, a alta concentração de glicose leva ao aumento de citocinas que estimulam a síntese da iNOS, principalmente mediada pela PKC e aldose redutase, promovendo expansão da matriz extracelular pela deposição de fibronectina (72).

Em células endoteliais, a Akt tem sido identificada como uma das responsáveis pela ativação da eNOS, independentemente da presença do cálcio (78), através da fosforilação da Serl177/1179. Feliers e cols. (79) descreveram aumento da fosforilação da tirosina em proteínas de córtex renal em camundongos geneticamente modificados, com diabetes $(\mathrm{db} / \mathrm{db})$, como a PI3K, Akt e ERKl/2.

Trabalhos in vitro têm demonstrado que a eNOS é regulada por uma via coordenada de fosforilação e desfosforilação de resíduos de aminoácidos, como a tirosina, serina e treonina (80). Por exemplo, a fosforilação da Ser1177/1179 leva à ativação da enzima NOS (81), enquanto que a fosforilação da Thr495 tem efeito oposto, desativando a NOS (80). No diabetes, várias vias de sinalização podem estar alteradas, influenciando a fosforilação da eNOS e, provavelmente, das demais isoformas da NOS. 
O aumento da expressão da iNOS relaciona-se de forma geral com o processo inflamatório que acompanha a nefropatia diabética, não tendo papel na hemodinâmica renal normal. Por outro lado, a eNOS, expressa nas células endoteliais glomerulares, além de contribuir de forma expressiva com o óxido nítrico para as funções renais normais, parece estar envolvida nos estágios iniciais da nefropatia. Tal controvérsia envolve o modelo e espécies experimentais estudados, o tempo do diabetes, controle metabólico, tratamento ou não com insulina e, principalmente, o método de avaliação do óxido nítrico ou da enzima NOS utilizados.

O papel do óxido nítrico na nefropatia diabética ainda não está claro. Sabe-se, entretanto, que está envolvido tanto na fase precoce quanto na tardia da doença renal. Chin e cols. (82) sugerem que esta aparente ambigüidade da ação do óxido nítrico deva-se a efeitos diferentes: o óxido nítrico teria um efeito direto induzindo a nitrosilação de proteínas envolvidas na proliferação celular, e um efeito indireto modulando a atividade $\mathrm{e} / \mathrm{ou}$ produção de outros mensageiros celulares, que por sua vez inibiriam a proliferação celular. Esta idéia sugere que o óxido nítrico em condições fisiológicas, na ausência de estímulo, não estaria envolvido na modulação do crescimento celular, ao passo que, na presença de estímulo, o aumento do óxido nítrico teria papel importante regulando o crescimento celular.

\section{EVENTOS MOLECULARES DA LESÃO RENAL ASSOCIADOS AO SISTEMA RENINA-ANGIOTENSINA}

O SRA vem sendo extensivamente estudado no diabetes, com alguns resultados conflitantes: aumento, diminuição ou não alteração do SRA (32). Estas discrepâncias podem ser explicadas devido à quantidade de fatores que influenciam o SRA e diferentes espécies e estágios da doença estudados (25), bem como o nível glicêmico estabelecido durante a doença (30).

Vários modelos de diabetes mostram que o nível renal de renina está elevado em relação aos níveis plasmáticos, sugerindo uma diminuição na liberação renal de renina na circulação (83). Estudos com micropunção mostram que a concentração de AII em diversos compartimentos intra-renais é muito maior do que a concentração sistêmica (84). Sendo assim, a atividade da renina plasmática pode não refletir corretamente a condição do SRA no rim (85).

Resultados obtidos de experimentos realizados em ratos diabéticos sugerem que o uso de um agente anti-hipertensivo específico (inibidores da ECA) pode preservar a função e a estrutura renal independente de seus outros efeitos na pressão sangüínea sistêmica (86). Nestes animais, o uso de agentes anti-hipertensivos convencionais preveniu parcialmente o desenvolvimento de glomeruloesclerose. Entretanto, o uso de captopril (inibidor da ECA) preveniu completamente o desenvolvimento de glomeruloesclerose. O possível mecanismo de proteção postulado seria a diminuição do efeito constritor tônico da AII na arteríola eferente, levando à diminuição da pressão intracapilar glomerular e ao bloqueio dos efeitos da AII sobre o crescimento celular e produção de matriz extracelular no rim (87).

Estudos recentes têm ampliado nosso conhecimento sobre eventos celulares mediados pela AII, sugerindo que seu papel biológico é tão amplo que vai do intracelular ao tecido ou ao sistema. Apesar da AII ser a substância ativa mais importante do SRA, outras angiotensinas produzidas têm ações específicas e, entre as melhores caracterizadas até o momento, incluem-se as angiotensinas III e IV e a angiotensina (1-7) (88). Todas estas angiotensinas podem ser produzidas a partir do mesmo precursor, o angiotensinogênio, por ação da renina e outras reações enzimáticas. Entre estas, a angiotensina (1-7) tem sido a mais estudada, geralmente apresentando efeitos opostos aos da AII, levando à vasodilatação mediada por óxido nítrico, potenciando o efeito hipotensor da bradicinina, facilitando o reflexo presso-receptor e participando do efeito antitrombótico de drogas como captopril e losartan (88).

Além das funções já caracterizadas, a AII vem sendo considerada como uma citocina multifuncional com propriedades não-hemodinâmicas, entre as quais a de fator de crescimento, de citocina pró-fibrinogênica e pró-inflamatória (89), e moduladora da resposta imunológica, como a quimiotaxia, a proliferação e a diferenciação de monócitos em macrófagos (90). De fato, a AII tem vários efeitos como induzir a adesão de leucócitos a células endoteliais, modular a expressão de e-selectina, uma molécula de adesão que está implicada no contato inicial entre leucócitos e endotélio e que desempenha um papel importante no processo de rolamento de leucócitos e, conseqüentemente, na inflamação (91).

No modelo de hipertensão induzido por altas concentrações plasmáticas de AII observam-se lesões teciduais decorrentes de seu efeito vasoconstritor, aumento da secreção de endotelinas e aldosterona e disfunção endotelial pela produção de estresse oxidativo, e pelo aumento da adesão de leucócitos às células endoteliais (92). Além disso, vários estudos demonstraram que o bloqueio da ação da AII previne a infla- 
mação, a expressão de MCP-1 e a infiltração de monócitos na parede arterial, assim como diminui a resposta inflamatória renal e a hipertensão arterial (93).

A maioria das ações clássicas da AII é mediada pelo receptor $\mathrm{AT}_{1}$ (94). Em ratos hipertensos transgênicos (com genes de renina e angiotensinogênio humanos), o tratamento com inibidores da enzima conversora ou com inibidores do receptor $\mathrm{AT}_{1}$ preveniu o desenvolvimento de albuminúria, recrutamento de monócitos e resposta vascular inflamatória (95). Demonstrou-se, também, que a estimulação de receptores $\mathrm{AT}_{1}$ provoca hipertrofia e aumento da síntese de matriz extracelular em células musculares lisas (96).

Amiri e cols. (97) demonstraram que a alta concentração de glicose potencializa os efeitos das AII na indução da ativação de JAK2 (fosforilação em resíduo de tirosina), o qual é acompanhado da fosforilação em tirosina e/ou serina de STAT1 e STAT3 em células mesangiais e células de musculatura lisa de vaso. A ativação de JAK2 é essencial para a indução da produção de colágeno tipo IV e proliferação induzida tanto pela alta concentração de glicose como também pela AII. Além disso, estudos desse mesmo grupo demonstraram que a ativação de JAK2 e STAT1 é um processo necessário para a indução da produção de TGF- $\beta 1$ e fibronectina estimulada pela alta concentração de glicose nas células mesangiais (98).

Banes e cols. (99) demonstraram que a alta concentração de glicose estimula a fosforilação de JAK2, STATl e STAT3 in vivo, e que a fosforilação destas proteínas era reduzida quando os ratos eram tratados com o inibidor de ATl (candesartan) e pelo inibidor de JAK2 (AG490). Em adição, este estudo demonstrou que em ratos diabéticos a administração de AG490 reduziu tanto a pressão sistólica quanto a excreção urinária de proteína (99). Esses resultados indicam uma importante relação entre a via de sinalização JAK/STAT, a indução da produção de proteínas de matriz e a proliferação de células mesangiais, eventos presentes na nefropatia diabética.

Recentemente, Banes-Berceli e cols. (100) demonstraram que a utilização de simvastatina suprimia os efeitos da alta concentração de glicose e da AII na ativação de JAK2, STAT1 e STAT3 em células mesangiais in vitro e em glomérulo de ratos diabéticos in vivo. Concomitantemente, este estudo mostrou que a simvastatina promovia a redução na produção de colágeno tipo IV em células mesangiais exposta a altas concentrações de glicose sozinha ou associada com AII, além de demonstrarem que o tratamento com a simvastatina em ratos diabéticos também reduzia a excreção de proteínas.

\section{EVENTOS MOLECULARES DA LESÃO RENAL ASSOCIADOS AOS LÍPIDES}

Desde Kimmelstiel \& Wilson (101), com a descrição da glomeruloesclerose nodular clássica e da presença de depósitos de lípides nos rins diabéticos, vários pesquisadores também já verificaram depósito de lípides em rins diabéticos de humanos ou animais experimentais, e estes pesquisadores propõem que depósito lipídico pode ser um fator na patogênese da doença renal diabética (102).

A alta concentração de glicose, AII, noradrenalina, bradicinina, TGF- $\beta$ e o PDGF podem ativar fosfolipases intracelulares (fosfolipase A2), promovendo a formação de ácido araquidônico, que pode ser metabolizado pelas ciclooxigenases, citocromos P-450 (CYP) e lipooxigenases (LO) (103). A metabolização do ácido araquidônico catalisado pelas CYP é uma importante via de formação de mediadores autócrinos e parácrinos de numerosos efeitos biológicos.

A ômega hidroxilação do ácido araquidônico gera níveis significativos de 20-ácido hidroxieicosatetraenóico (20-HETE) e ácido epoxieicosatrienóico estéreo específico (EETs) em vários tecidos, principalmente em vasculatura, pulmão e túbulos renais (104). O 20-HETE apresenta vários efeitos; em célula muscular lisa inibe a condutância de cálcio ativado pelos canais de potássio, resultando em despolarização das células lisas (105).

$\mathrm{O}$ aumento na expressão e função de CYP subtipo 4A (CYP4A) foi documentado em rim de animais diabéticos (104). Zangar \& Novak (106) demonstraram que a elevação intracelular de ácidos graxos durante o diabetes contribuía com os efeitos no CYP4A. Em adição a esses achados, Kroetz e cols. (104) demonstraram que a ativação de PPAR $\alpha$ é um passo necessário nos efeitos da diabetes para a transcrição de CYP4A. Kroetz \& Xu (107) sugerem que baixas produções renais de 20-HETE em diabetes podem promover efeitos protetores por reduzir as ações vasoconstritoras desses eicosanóides.

As LO são enzimas da família que não contêm o grupo ferro no heme (nonbeme iron-containing enzymes) e que insere o oxigênio molecular nos ácidos graxos poliinsaturados (108). Kang e cols. (109) demonstraram que a expressão do gene da enzima 12/15 lipoxigenase e sua proteína aumentavam pela elevação da concentração de glicose em células mesangiais. Além disso, demonstraram que a expressão de fibronectina do glomérulo de ratos estava associada com o aumento da expressão de 12-lipooxigenase, implicando essa enzima na patogênese da nefropatia diabética. 
Reddy e cols. (110) demonstraram que lípides oxidados, tais como o $12(S)$-ácido hidroxieicosatetraenóico [12(S)-HETE], podem diretamente induzir em células mesangiais hipertrofia e a produção de proteínas de matriz extracelular, com efeito similar ao da AII. Além disso, esses pesquisadores demonstraram que tal efeito acontece porque o $12(\mathrm{~S})$-HETE é capaz de ativar a p38MAPK e o fator de transcrição nuclear CREB (cAMP-responsive element-binding protein), sugerindo dessa forma que a ativação de 12lipooxigenase e produção de $12(S)$-HETE podem estar envolvidos em múltiplos eventos relacionados com o desenvolvimento da nefropatia diabética.

Sun e cols. (111), por sua vez, usando o modelo estreptozotocina para indução de diabetes em ratos, demonstraram que os depósitos de lípides ocorrem sem qualquer alteração nos níveis séricos de colesterol ou triglicerídeos. Além disso, verificaram que o aumento renal dos depósitos de lípides estava correlacionado com o aumento da expressão da proteína ligante do elemento responsível a esteróides - 1 (SREBP-1), o que resultava num aumento da expressão da enzima-chave na síntese de ácido graxo (ácido graxo sintase) e num aumento no acúmulo de triglicerídeos. Neste mesmo estudo, os autores verificaram que o acúmulo de triglicerídeos e o aumento da expressão do SREBP-1 induziam aumento da expressão do TGF- $\beta 1$ e do fator de crescimento endotelial vascular (VEGF), como também aumento de colágeno e de fibronectina, o que pode resultar na glomeruloesclerose e na proteinúria.

A significância clínica do aumento dos conteúdos de lípides nas doenças renais está no desenvolvimento da esclerose glomerular, que pode progredir para a insuficiência renal (112). O efeito potencial das triglicérides como mediador de doenças renais diabéticas é ilustrado nos estudos com pacientes diabéticos que fazem tratamentos com agonistas de PPAR $\alpha$ e que apresentam redução nas concentrações séricas de triglicérides, resultando também em uma significante redução para proteinúria, um marcador clínico para a injúria renal diabética (113).

Os PPARs (receptores ativados por proliferadores de peroxissoma) são fatores de transcrição de uma superfamília de receptores nucleares, caracterizados por seu padrão de distribuição nos tecidos e por sua função metabólica, onde os membros da família dos PPARs desempenham papel-chave no metabolismo de lipídeos $(104,114,115)$.

Como mencionado anteriormente, o TGF- $\beta 1$ modula o desenvolvimento da glomeruloesclerose pela estimulação da produção das proteínas de matriz extracelular, pelas células mesangiais. Wilmer e cols.
(116) demonstraram que a estimulação do PPAR $\alpha$ antagonisa a expressão do TGF- $\beta 1$ em células mesangiais humanas. Utilizando a técnica de RT-PCR e imuno-histoquímica, estes autores demonstraram que as células mesangiais apresentam PPAR $\alpha$ e que os ligantes de PPARa (Wyl4643 ou clofibrato) podem atuar na modulação do TGF- $\beta 1$.

Routh e cols. (117) demonstraram, através de RT-PCR, a presença de PPAR $\alpha$ e PPAR $\gamma$ em células mesangiais de ratos, e que o tratamento com troglitazona resulta em uma significante redução no colágeno tipo I e uma pequena redução na produção de laminina, entretanto o tratamento com clofibrato não apresentou nenhum efeito na síntese de laminina, mas conseguiram induzir uma redução na síntese de colágeno tipo I nas células em cultura com alta concentração de glicose.

De forma similar, Tsuchiya e cols. (118) demonstraram a presença do RNAm do PPAR $\gamma$ em célula mesangial isolada de rato, e verificaram que a troglitazona inibe a proliferação e induz apoptose nessas células, sugerindo um efeito direto na função renal. Parameswaran e cols. (119) também demonstraram que outro membro da família das tiazolidinedionas, a ciglitazona, induz apoptose em células mesangiais e causa significante redução no crescimento celular.

\section{CONSIDERAÇÕES FINAIS}

Os mecanismos patogenéticos associados à glomerulopatia diabética ainda não estão totalmente esclarecidos, porque muitos dos eventos moleculares associados à lesão renal ainda precisam ser desvendados. Contudo, sabe-se que as alterações na viabilidade das células mesangiais e nos depósitos de matriz, estimuladas direta ou indiretamente pela elevação da glicose, estão relacionadas com a lesão renal associada ao diabetes. Além disso, a literatura relata que não existe uma estratégia terapêutica específica que possa ser recomendada para a nefropatia diabética, sendo possível apenas controlar parcialmente os fatores associados à gênese e à progressão da glomerulopatia diabética. Dessa forma, as pesquisas direcionadas à compreensão dos mecanismos moleculares da glomerulopatia diabética poderão resultar em terapias mais apropriadas a serem testadas e utilizadas posteriormente.

\section{AGRADECIMENTOS}

Nosso grupo recebe suporte financeiro da FAPESP, CNPq, CAPES e Pronex. 


\section{REFERÊNCIAS}

1. King $\mathrm{H}$, Aubert RE, Herman WH. Global burden of diabetes, 1995-2025: prevalence, numerical estimates, and projections. Diabetes Care 1998;21(9):1414-31.

2. Malerbi DA, Franco LJ. Multicenter study of the prevalence of diabetes mellitus and impaired glucose tolerance in the urban Brazilian population aged 30-69 yr. The Brazilian Cooperative Group on the Study of Diabetes Prevalence. Diabetes Care 1992;15(11):1509-16.

3. Schaan BD, Harzheim E, Gus I. [Cardiac risk profile in diabetes mellitus and impaired fasting glucose]. Rev Saúde Pública 2004;38(4):529-36.

4. Kannel WB, McGee DL. Diabetes and cardiovascular disease. The Framingham study. JAMA 1979;241(19):2035-8.

5. Bruno RM, Gross JL. Prognostic factors in Brazilian diabetic patients starting dialysis: a 3.6-year follow-up study. J Diabetes Complications 2000;14(5):266-71.

6. DCCT. The effect of intensive treatment of diabetes on the development and progression of long-term complications in insulin-dependent diabetes mellitus. The Diabetes Control and Complications Trial Research Group. N Engl J Med 1993:329(14):977-86

7. Hovind $P$, Tarnow $L$, Parving $H H$. Remission and regression of diabetic nephropathy. Curr Hypertens Rep 2004;6(5):377-82.

8. Rossing $P$. The changing epidemiology of diabetic microangiopathy in type 1 diabetes. Diabetologia 2005;48(8):1439-44.

9. Zintzaras E, Stefanidis I. Association between the GLUT1 gene polymorphism and the risk of diabetic nephropathy: a meta-analysis. J Hum Genet 2005;50(2):84-91.

10. Parving HH, Andersen AR, Smidt UM, Hommel E, Mathiesen ER, Svendsen PA. Effect of antihypertensive treatment on kidney function in diabetic nephropathy. Br Med J (Clin Res Ed) 1987;294(6585):1443-7.

11. Brownlee $M$. The pathobiology of diabetic complications: a unifying mechanism. Diabetes 2005;54(6):1615-25.

12. Striker GE, Soderland C, Bowen-Pope DF, Gown AM, Schmer G, Johnson A, et al. Isolation, characterization, and propagation in vitro of human glomerular endothelial cells. $\mathbf{J}$ Exp Med 1984;160(1):323-8.

13. Kashtan CE. Clinical and molecular diagnosis of Alport syndrome. Proc Assoc Am Physicians 1995;107(3):306-13.

14. Haralson MA, Jacobson HR, Hoover RL. Collagen polymorphism in cultured rat kidney mesangial cells. Lab Invest 1987;57(5):513-23.

15. Doi T, Striker LJ, Kimata K, Peten EP, Yamada Y, Striker GE. Glomerulosclerosis in mice transgenic for growth hormone. Increased mesangial extracellular matrix is correlated with kidney mRNA levels. J Exp Med 1991;173(5):1287-90.

16. Yang CW, Striker GE, Chen WY, Kopchick JJ, Striker LJ. Differential expression of glomerular extracellular matrix and growth factor mRNA in rapid and slowly progressive glomerulosclerosis: studies in mice transgenic for native or mutated growth hormone. Lab Invest 1997;76(4):467-76.

17. Ayo SH, Radnik R, Garoni JA, Troyer DA, Kreisberg JI. High glucose increases diacylglycerol mass and activates protein kinase $C$ in mesangial cell cultures. Am J Physiol 1991;261(4 Pt 2):F571-7.

18. Studer RK, Craven PA, DeRubertis FR. Role for protein kinase $C$ in the mediation of increased fibronectin accumulation by mesangial cells grown in high-glucose medium. Diabetes 1993;42(1):118-26.

19. Gould GW, Holman GD. The glucose transporter family: structure, function and tissue-specific expression. Biochem J 1993;295(Pt 2):329-41.

20. Henry DN, Busik JV, Brosius FC, 3rd, Heilig CW. Glucose transporters control gene expression of aldose reductase, PKC $\alpha$, and GLUT1 in mesangial cells in vitro. Am J Physiol 1999;277(1 Pt 2):F97-104.

21. Schaan BD, Irigoyen MC, Bertoluci MC, Lima NG, Passaglia J, Hermes $E$, et al. Increased urinary TGF- $\beta 1$ and cortical renal GLUT1 and GLUT2 levels: additive effects of hypertension and diabetes. Nephron Physiol 2005;100(3):p43-50.
22. Vestri S, Okamoto MM, de Freitas HS, Santos RA, Nunes MT, Morimatsu $\mathrm{M}$, et al. Changes in sodium or glucose filtration rate modulate expression of glucose transporters in renal proximal tubular cells of rat. J Membr Biol 2001;182(2):105-12.

23. Zhang J, Liu Z, Liu D, Li L. Identification of glucose transporter-1 and its functional assay in mouse glomerular mesangial cells cultured in vitro. Chin Med Sci J 2001;16(1):35-9.

24. Li Y, Liu Z, Liu D, Zhang J, Chen Z, Li L. Identification and function of glucose transporter 1 in human mesangial cells. Chin Med J (Engl) 2001;114(8):824-8.

25. Leehey DJ, Singh AK, Alavi N, Singh R. Role of angiotensin II in diabetic nephropathy. Kidney Int Suppl 2000;77:S93-8.

26. Nose A, Mori Y, Uchiyama-Tanaka Y, Kishimoto N, Maruyama K, Matsubara $\mathrm{H}$, et al. Regulation of glucose transporter (GLUT1) gene expression by angiotensin II in mesangial cells: involvement of $\mathrm{HB}-\mathrm{EGF}$ and $\mathrm{EGF}$ receptor transactivation. Hypertens Res 2003;26(1):67-73.

27. Uchiyama-Tanaka Y, Matsubara H, Nozawa Y, Murasawa S, Mori $Y$, Kosaki A, et al. Angiotensin II signaling and HB-EGF shedding via metalloproteinase in glomerular mesangial cells. Kidney Int 2001;60(6):2153-63.

28. Andrade MC, Quinto BM, Carmona AK, Ribas OS, Boim MA, Schor $\mathrm{N}$, et al. Purification and characterization of angiotensin I-converting enzymes from mesangial cells in culture. J Hypertens 1998;16(12 Pt 2):2063-74.

29. Vidotti DB, Casarini DE, Cristovam PC, Leite CA, Schor N, Boim MA. High glucose concentration stimulates intracellular renin activity and angiotensin II generation in rat mesangial cells. Am J Physiol Renal Physiol 2004;286(6):F1039-45.

30. Burns KD. Angiotensin II and its receptors in the diabetic kidney. Am J Kidney Dis 2000;36(3):449-67.

31. Burns KD, Homma $T$, Harris RC. The intrarenal reninangiotensin system. Semin Nephrol 1993;13(1):13-30.

32. Wolf G, Ziyadeh FN. The role of angiotensin II in diabetic nephropathy: emphasis on nonhemodynamic mechanisms. Am J Kidney Dis 1997;29(1):153-63.

33. Quinto BM, Juliano MA, Hirata I, Carmona AK, Juliano L, Casarini DE. Specificity comparison of a serine endopeptidase (SH1) and a serine thiol endopeptidase (STH2) purified from human urine. Int J Biochem Cell Biol 2004;36(10):1933-44.

34. Camargo de Andrade MC, Di Marco GS, de Paulo Castro Teixeira V, Mortara RA, Sabatini RA, Pesquero JB, et al. Expression and localization of $\mathrm{N}$-domain ANG I-converting enzymes in mesangial cells in culture from spontaneously hypertensive rats. Am J Physiol Renal Physiol 2006;290(2):F364-75.

35. Lovett DH, Szamel M, Ryan JL, Sterzel RB, Gemsa D, Resch K. Interleukin 1 and the glomerular mesangium. I. Purification and characterization of a mesangial cell-derived autogrowth factor. J Immunol 1986;136(10):3700-5.

36. Conti FG, Striker LJ, Lesniak MA, MacKay K, Roth J Striker GE. Studies on binding and mitogenic effect of insulin and insulin-like growth factor I in glomerular mesangial cells. Endocrinology 1988;122(6):2788-95.

37. Ardaillou R, Chansel D, Chatziantoniou C, Dussaule JC. Mesangial AT1 receptors: expression, signaling, and regulation. J Am Soc Nephrol 1999;10(suppl 11):S40-6.

38. Simonson MS, Wann S, Mene P, Dubyak GR, Kester M, Nakazato $Y$, et al. Endothelin stimulates phospholipase C, $\mathrm{Na}^{+} / \mathrm{H}^{+}$exchange, c-fos expression, and mitogenesis in rat mesangial cells. J Clin Invest 1989;83(2):708-12.

39. Castellot JJ, Jr., Hoover RL, Karnovsky MJ. Glomerular endothelial cells secrete a heparin-like inhibitor and a peptide stimulator of mesangial cell proliferation. Am J Pathol 1986;125(3):493-500.

40. Righetti AE, Boer-Lima PA, Lopes de Faria JB. The presence of genetic hypertension stimulates early renal accumulation of fibronectin in experimental diabetes mellitus. Diabetologia $2001 ; 44(11): 2088-91$.

41. Ziyadeh FN, Cohen MP. Effects of glycated albumin on mesangial cells: evidence for a role in diabetic nephropathy. Mol Cell Biochem 1993:125(1):19-25.

42. Singh R, Alavi N, Singh AK, Leehey DJ. Role of angiotensin II in glucose-induced inhibition of mesangial matrix degradation. Diabetes 1999:48(10):2066-73. 
43. Isono M, Mogyorosi A, Han DC, Hoffman BB, Ziyadeh FN. Stimulation of TGF- $\beta$ type II receptor by high glucose in mouse mesangial cells and in diabetic kidney. Am J Physiol Renal Physiol 2000;278(5):F830-8.

44. Singh R, Singh AK, Alavi N, Leehey DJ. Mechanism of increased angiotensin II levels in glomerular mesangial cells cultured in high glucose. J Am Soc Nephrol 2003; 14(4):873-80.

45. Okado $T$, Terada $Y$, Tanaka $H$, Inoshita $S$, Nakao A, Sasaki S. Smad7 mediates transforming growth factor-beta-induced apoptosis in mesangial cells. Kidney Int 2002;62(4):1178-86.

46. Lagranha C, Doi S, Pithon-Curi T, Suzuki K, Kohn L, Sellitti D. TGF- $\beta$ 1-like activity of thyroglobulin in mouse mesangial cells. J Am Soc Nephrol 2002;13:77A-B.

47. Kim YS, Kim BC, Song CY, Hong HK, Moon KC, Lee HS. Advanced glycosylation end products stimulate collagen mRNA synthesis in mesangial cells mediated by protein kinase $\mathrm{C}$ and transforming growth factor-beta. $\mathbf{J}$ Lab Clin Med 2001;138(1):59-68.

48. Singh AK, Gudehithlu KP, Pegoraro AA, Singh GK, Basheerudin K, Robey RB, et al. Vascular factors altered in glucose-treated mesangial cells and diabetic glomeruli. Changes in vascular factors impair endothelial cell growth and matrix. Lab Invest 2004;84(5):597-606.

49. Way KJ, Katai N, King GL. Protein kinase C and the development of diabetic vascular complications. Diabet Med 2001;18(12):945-59.

50. Seshiah PN, Weber DS, Rocic P, Valppu L, Taniyama Y, Griendling KK. Angiotensin II stimulation of NAD(P)H oxidase activity: upstream mediators. Circ Res 2002:91(5):406-13.

51. Mene P, Pugliese G, Pricci F, Di Mario U, Cinotti GA, Pugliese $\mathrm{F}$. High glucose level inhibits capacitative $\mathrm{Ca}^{2+}$ influx in cultured rat mesangial cells by a protein kinase C-dependent mechanism. Diabetologia 1997;40(5):521-7.

52. Hua H, Munk S, Goldberg H, Fantus IG, Whiteside Cl. High glucose-suppressed endothelin-1 $\mathrm{Ca}^{2+}$ signaling via NADPH oxidase and diacylglycerol-sensitive protein kinase $\mathrm{C}$ isozymes in mesangial cells. J Biol Chem 2003;278(36):33951-62.

53. Xu D, Kyriakis JM. Phosphatidylinositol 3'-kinase-dependent activation of renal mesangial cell Ki-Ras and ERK by advanced glycation end products. J Biol Chem 2003;278(41):39349-55.

54. Katso $R$, Okkenhaug $K$, Ahmadi $K$, White $S$, Timms J, Waterfield MD. Cellular function of phosphoinositide 3-kinases: implications for development, homeostasis, and cancer. Annu Rev Cell Dev Biol 2001;17:615-75.

55. Rodriguez-Pena AB, Santos E, Arevalo M, Lopez-Novoa JM. Activation of small GTPase Ras and renal fibrosis. J Nephrol 2005;18(3):341-9.

56. Danesh FR, Sadeghi MM, Amro N, Philips C, Zeng L, Lin S, et al. 3-hydroxy-3-methylglutaryl CoA reductase inhibitors prevent high glucose-induced proliferation of mesangial cells via modulation of Rho GTPase/ p21 signaling pathway: Implications for diabetic nephropathy. Proc Natl Acad Sci U S A 2002;99(12):8301-5.

57. Padival AK, Hawkins KS, Huang C. High glucose-induced membrane translocation of PKC betal is associated with Arf6 in glomerular mesangial cells. Mol Cell Biochem 2004;258(1-2):129-35.

58. Lin S, Sahai A, Chugh SS, Pan X, Wallner El, Danesh FR, et al. High glucose stimulates synthesis of fibronectin via a novel protein kinase C, Rap1b, and B-Raf signaling pathway. J Biol Chem 2002;277(44):41725-35.

59. Schnaper HW, Hayashida T, Hubchak SC, Poncelet AC. TGF- $\beta$ signal transduction and mesangial cell fibrogenesis. Am $\mathbf{J}$ Physiol Renal Physiol 2003;284(2):F243-52.

60. Hubchak SC, Runyan CE, Kreisberg JI, Schnaper HW. Cytoskeletal rearrangement and signal transduction in TGFbeta1-stimulated mesangial cell collagen accumulation. $\boldsymbol{J}$ Am Soc Nephrol 2003;14(8):1969-80.

61. Mohaupt M, SchoeckImann HO, Schulze-Lohoff E, Sterzel RB. Altered nitric oxide production and exogenous nitric oxide do not affect the proliferation of rat mesangial cells. J Hypertens 1994;12(4):401-8.
62. Haas CS, Schocklmann HO, Lang S, Kralewski M, Sterzel RB. Regulatory mechanism in glomerular mesangial cell proliferation. J Nephrol 1999;12(6):405-15.

63. Komers R, Anderson S. Paradoxes of nitric oxide in the diabetic kidney. Am J Physiol Renal Physiol 2003;284(6):F1121-37

64. Moncada S, Palmer RM, Higgs EA. Nitric oxide: physiology, pathophysiology, and pharmacology. Pharmacol Rev 1991;43(2):109-42.

65. Cattell V. Nitric oxide and glomerulonephritis. Semin Nephrol 1999;19(3):277-87

66. Nath AK, Madri JA. The roles of nitric oxide in murine cardiovascular development. Dev Biol 2006;292(1):25-33.

67. Araujo M, Welch WJ. Oxidative stress and nitric oxide in kidney function. Curr Opin Nephrol Hypertens 2006;15(1):72-7.

68. Brune B, von Knethen A, Sandau KB. Transcription factors p53 and HIF- $1 \alpha$ as targets of nitric oxide. Cell Signal 2001;13(8):525-33.

69. Erwin PA, Lin AJ, Golan DE, Michel T. Receptor-regulated dynamic S-nitrosylation of endothelial nitric-oxide synthase in vascular endothelial cells. J Biol Chem 2005;280(20):19888-94.

70. Peppa M, Vlassara H. Advanced glycation end products and diabetic complications: a general overview. Hormones (Athens Greece) 2005;4(1):28-37

71. Chang PC, Chen TH, Chang CJ, Hou CC, Chan P, Lee HM. Advanced glycosylation end products induce inducible nitric oxide synthase (iNOS) expression via a p38 MAPK-dependent pathway. Kidney Int 2004;65(5):1664-75.

72. Noh $\mathrm{H}, \mathrm{Ha} \mathrm{H}, \mathrm{Yu}$ MR, Kang SW, Choi KH, Han DS, et al. High glucose increases inducible NO production in cultured rat mesangial cells. Possible role in fibronectin production. Nephron 2002;90(1):78-85.

73. Derubertis FR, Craven PA. Activation of protein kinase $\mathrm{C}$ in glomerular cells in diabetes. Mechanisms and potential links to the pathogenesis of diabetic glomerulopathy. Diabetes 1994:43(1):1-8.

74. Veelken R, Hilgers KF, Hartner A, Haas A, Bohmer KP, Sterzel RB. Nitric oxide synthase isoforms and glomerular hyperfiltration in early diabetic nephropathy. J Am Soc Nephrol 2000;11(1):71-9.

75. Khamaisi M, Keynan S, Bursztyn M, Dahan R, Reinhartz E, Ovadia $\mathrm{H}$, et al. Role of renal nitric oxide synthase in diabetic kidney disease during the chronic phase of diabetes. Nephron Physiol 2006;102(3-4):p72-80.

76. Hirata K, Kuroda R, Sakoda T, Katayama M, Inoue N, Suematsu $\mathrm{M}$, et al. Inhibition of endothelial nitric oxide synthase activity by protein kinase C. Hypertension 1995;25(2):180-5.

77. Michell BJ, Chen Zp, Tiganis T, Stapleton D, Katsis F, Power DA, et al. Coordinated control of endothelial nitric-oxide synthase phosphorylation by protein kinase $\mathrm{C}$ and the cAMP-dependent protein kinase. J Biol Chem 2001;276(21):17625-8.

78. Fulton D, Gratton JP, McCabe TJ, Fontana J, Fujio Y, Walsh $\mathrm{K}$, et al. Regulation of endothelium-derived nitric oxide production by the protein kinase Akt. Nature 1999;399 (6736):597-601.

79. Feliers D, Duraisamy S, Faulkner JL, Duch J, Lee AV, Abboud $\mathrm{HE}$, et al. Activation of renal signaling pathways in $\mathrm{db} / \mathrm{db}$ mice with type 2 diabetes. Kidney Int 2001;60(2):495-504.

80. Fleming I, Fisslthaler B, Dimmeler S, Kemp BE, Busse R. Phosphorylation of $\mathrm{Thr}(495)$ regulates $\mathrm{Ca}^{2+}$ /calmodulindependent endothelial nitric oxide synthase activity. Circ Res 2001;88(11):E68-75.

81. Harris MB, Ju H, Venema VJ, Liang $H$, Zou R, Michell BJ, et al. Reciprocal phosphorylation and regulation of endothelial nitric-oxide synthase in response to bradykinin stimulation. $\mathbf{J}$ Biol Chem 2001;276(19):16587-91.

82. Chin TY, Lin YS, Chueh SH. Antiproliferative effect of nitric oxide on rat glomerular mesangial cells via inhibition of mitogen-activated protein kinase. Eur J Biochem 2001;268(24):6358-68.

83. Kennefick TM, Anderson S. Role of angiotensin II in diabetic nephropathy. Semin Nephrol 1997;17(5):441-7.

84. Seikaly MG, Arant BS, Jr., Seney FD, Jr. Endogenous angiotensin concentrations in specific intrarenal fluid compartments of the rat. J Clin Invest 1990;86(4):1352-7. 
85. Amemiya S, Ishihara T, Higashida K, Kusano S, Ohyama K, Kato K. Altered synthesis of renin in patients with insulin-dependent diabetes: plasma prorenin as a marker predicting the evolution of nephropathy. Diabetes Res Clin Pract 1990;10(2):115-22.

86. Anderson S, Rennke HG, Garcia DL, Brenner BM. Short and long term effects of antihypertensive therapy in the diabetic rat. Kidney Int 1989;36(4):526-36.

87. Fogo AB. Mesangial matrix modulation and glomerulosclerosis. Exp Nephrol 1999:7(2):147-59.

88. Santos RA, Campagnole-Santos MJ, Andrade SP. Angiotensin-(1-7): an update. Regul Pept 2000;91(1-3):45-62.

89. Wolf G, Butzmann U, Wenzel UO. The renin-angiotensin system and progression of renal disease: from hemodynamics to cell biology. Nephron Physiol 2003;93(1):P3-13.

90. Ruiz-Ortega M, Lorenzo O, Ruperez M, Esteban V, Suzuki Y, Mezzano S, et al. Role of the renin-angiotensin system in vascular diseases: expanding the field. Hypertension $2001 ; 38(6): 1382-7$.

91. Mai M, Hilgers KF, Geiger $\mathrm{H}$. Experimental studies on the role of intercellular adhesion molecule-1 and lymphocyte function-associated antigen-1 in hypertensive nephrosclerosis. Hypertension 1996;28(6):973-9.

92. Nagy L, Thomazy VA, Davies PJ. A transgenic mouse model for the study of apoptosis during limb development. Cell Death Differ 1998;5(1):126.

93. Beswick RA, Zhang H, Marable D, Catravas JD, Hill WD, Webb $\mathrm{RC}$. Long-term antioxidant administration attenuates mineralocorticoid hypertension and renal inflammatory response. Hypertension 2001;37(2 Part 2):781-6.

94. Matsusaka T, Fogo A, Ichikawa I. Targeting the genes of angiotensin receptors. Semin Nephrol 1997;17(5):396-403.

95. Mervaala EM, Muller DN, Park JK, Schmidt F, Lohn M, Breu $\mathrm{V}$, et al. Monocyte infiltration and adhesion molecules in a rat model of high human renin hypertension. Hypertension 1999;33(1 Pt 2):389-95.

96. Kato $H$, Suzuki $H$, Tajima $S$, Ogata $Y$, Tominaga $T$, Sato $A$, et al. Angiotensin II stimulates collagen synthesis in cultured vascular smooth muscle cells. J Hypertens 1991;9(1):17-22.

97. Amiri F, Shaw S, Wang X, Tang J, Waller JL, Eaton DC, et al. Angiotensin II activation of the JAK/STAT pathway in mesangial cells is altered by high glucose. Kidney Int 2002;61(5):1605-16.

98. Wang X, Shaw S, Amiri F, Eaton DC, Marrero MB. Inhibition of the JAK/STAT signaling pathway prevents the high glucose-induced increase in TGF- $\beta$ and fibronectin synthesis in mesangial cells. Diabetes 2002;51(12):3505-9.

99. Banes AK, Shaw S, Jenkins J, H, Amiri F, Pollock DM, et al. Angiotensin II blockade prevents hyperglycemia-induced activation of JAK and STAT proteins in diabetic rat kidney glomeruli. Am J Physiol Renal Physiol 2004;286(4):F653-9.

100.Banes-Berceli AK, Shaw S, Ma G, Brands M, Eaton DC, Stern DM, et al. Effect of Simvastatin on High Glucose-and Angiotensin II-Induced Activation of the JAK/STAT Pathway in Mesangial Cells. Am J Physiol Renal Physiol 2006;291(1):F116-21.

101. Kimmelsteil P, Wilson C. Intercapillary lesion in the glomeruli of the kidney. Am J Physiol 1936:12:83-105.

102.Lee HS, Lee JS, Koh HI, Ko KW. Intraglomerular lipid deposition in routine biopsies. Clin Nephrol 1991;36(2):67-75.

103. Mukherjee AB, Miele L, Pattabiraman N. Phospholipase A2 enzymes: regulation and physiological role. Biochem Pharmacol 1994;48(1):1-10.

104.Kroetz DL, Yook P, Costet P, Bianchi P, Pineau T. Peroxisome proliferator-activated receptor alpha controls the hepatic CYP4A induction adaptive response to starvation and diabetes. J Biol Chem 1998;273(47):31581-9.
105.Zou AP, Fleming JT, Falck JR, Jacobs ER, Gebremedhin D, Harder DR, et al. 20-HETE is an endogenous inhibitor of the large-conductance $\mathrm{Ca}^{2+}$-activated $\mathrm{K}^{+}$channel in renal arterioles. Am J Physiol 1996;270(1 Pt 2):R228-37.

106.Zangar RC, Novak RF. Effects of fatty acids and ketone bodies on cytochromes $\mathrm{P} 450$ 2B, 4A, and 2E1 expression in primary cultured rat hepatocytes. Arch Biochem Biophys 1997;337(2):217-24.

107. Kroetz DL, Xu F. Regulation and inhibition of arachidonic acid omega-hydroxylases and 20-HETE formation. Annu Rev Pharmacol Toxicol 2005;45:413-38.

108. Yamamoto S. Mammalian lipoxygenases: molecular structures and functions. Biochem Biophys Acta 1992;1128(23):117-31.

109.Kang SW, Adler SG, Nast CC, LaPage J, Gu JL, Nadler JL, et al. 12-lipoxygenase is increased in glucose-stimulated mesangial cells and in experimental diabetic nephropathy. Kidney Int 2001;59(4):1354-62.

110.Reddy MA, Adler SG, Kim YS, Lanting L, Rossi J, Kang SW, et al. Interaction of MAPK and 12-lipoxygenase pathways in growth and matrix protein expression in mesangial cells. Am J Physiol Renal Physiol 2002;283(5):F985-94.

111.Sun L, Halaihel N, Zhang W, Rogers T, Levi M. Role of sterol regulatory element-binding protein 1 in regulation of renal lipid metabolism and glomerulosclerosis in diabetes mellitus. J Biol Chem 2002;277(21):18919-27.

112. Obara K, Saito T, Sato H, Ogawa M, Igarashi Y, Yoshinaga K. Renal histology in two adult patients with type I glycogen storage disease. Clin Nephrol 1993;39(2):59-64.

113. Fried LF, Orchard TJ, Kasiske BL. Effect of lipid reduction on the progression of renal disease: a meta-analysis. Kidney Int 2001;59(1):260-9.

114. Nasrallah R, Hebert RL. Prostacyclin signaling in the kidney: implications for health and disease. Am J Physiol Renal Physiol 2005;289(2):F235-46.

115. Huang TH, Peng G, Li GQ, Yamahara J, Roufogalis BD, Li Y Salacia oblonga root improves postprandial hyperlipidemia and hepatic steatosis in Zucker diabetic fatty rats: activation of PPAR $\alpha$. Toxicol Appl Pharmacol 2006;210(3):225-35.

116.Wilmer WA, Dixon CL, Hebert C, Lu L, Rovin BH. PPAR $\alpha$ ligands inhibit H2O2-mediated activation of transforming growth factor-beta1 in human mesangial cells. Antioxid Redox Signal 2002;4(6):877-84.

117. Routh RE, Johnson JH, McCarthy KJ. Troglitazone suppresses the secretion of type I collagen by mesangial cells in vitro. Kidney Int 2002;61(4):1365-76

118.Tsuchiya T, Shimizu H, Shimomura K, Mori M. Troglitazone inhibits isolated cell proliferation, and induces apoptosis in isolated rat mesangial cells. Am J Nephrol 2003;23(4):222-8.

119. Parameswaran N, Hall CS, Bomberger JM, Sparks HV, Jump DB, Spielman WS. Negative growth effects of ciglitazone on kidney interstitial fibroblasts: role of PPAR $\gamma$. Kidney Blood Press Res 2003;26(1):2-9.

Endereço para correspondência:

Maria Claudia Irigoyen

Laboratório de Hipertensão Experimental

Instituto do Coração-FMUSP

Av. Dr. Enéas de Carvalho Aguiar 44

05403-000 São Paulo, SP

Fax: (1 1) 3085-7887 